\title{
Comparison of ovarian torsion between pregnant and non-pregnant women at reproductive ages: sonographic and pathological findings
}

\author{
Jie-Ling Feng ${ }^{1 \#}$, Ju Zheng $^{1 \#}$, Ting Lei ${ }^{1}$, Yong-Jian $\mathrm{Xu}^{2}$, Hui Pang ${ }^{3}$, Hong-Ning Xie ${ }^{1}$ \\ ${ }^{1}$ Department of Ultrasonic Medicine, Fetal Medical Center, ${ }^{2}$ Center for Information Technology \& Statistics, ${ }^{3}$ Department of Medical Records \\ Management, First Affiliated Hospital of Sun Yat-sen University, Guangzhou 510080, China
}

\#These authors contributed equally to this work.

Correspondence to: Hong-Ning Xie. Department of Ultrasonic Medicine, Fetal Medical Center, First Affiliated Hospital of Sun Yat-sen University, Zhongshan Er Road \#58, Guangzhou 510080, Guangdong, China. Email: hongning_x@126.com.

Background: Differences in the ultrasonographic features and histological diagnosis of ovarian torsion in pregnant and non-pregnant women have not been defined. A better characterization of these features may help improve the accuracy of preoperative diagnosis. The present study aimed to compare the clinical characteristics, sonographic findings, operative procedures, and histological spectrum of ovarian torsion in pregnant and non-pregnant women.

Methods: This was a retrospective investigation of female patients at reproductive age with ovarian torsion between January 2010 and May 2017. Each patient received a detailed preoperative ultrasound, and the diagnosis was confirmed by surgery. The clinical characteristics, ultrasonic features, operative procedures, and histological diagnosis of ovarian torsion were retrieved from medical records and were compared in nonpregnant and pregnant patients according to the method of conception.

Results: The overall preoperative ultrasonic detection rate of ovarian torsion was 0.84 , which was significantly different between pregnant and non-pregnant women. The presence of ovarian edema and abnormal adnexal positions also differed between pregnant and non-pregnant women. The ultrasonic features were not significantly different between the two pregnant sub-groups. The most common histologic diagnoses in the pregnant group and the non-pregnant group were a normal ovary and teratoma, respectively. The incidence of ovarian neoplasm was significantly lower in pregnant women. There were significant differences in the surgical procedures between the groups based on neoplastic or non-neoplastic lesions.

Conclusions: Ovarian edema, absence/decreased blood flow in the ovary, and the whirlpool sign were reliable ultrasonic markers for ovarian torsion at reproductive ages. The preoperative ultrasonic detection rate of ovarian torsion was higher in pregnant women, and ovarian edema was more common. The clinical features of ovarian torsion in pregnant women were similar, independent of the method of conception. In women with ovarian torsion, the incidence of non-neoplastic lesions was more frequent in pregnant women, whereas neoplastic lesions were more common in non-pregnant women. Ultrasonography provides useful parameters for the preclinical diagnosis of ovarian torsion to improve patient management.

Keywords: Ovarian torsion; pathology; pregnancy; ultrasonography

Submitted Mar 26, 2019. Accepted for publication Oct 28, 2019.

doi: $10.21037 /$ qims.2019.11.06

View this article at: http://dx.doi.org/10.21037/qims.2019.11.06 


\section{Introduction}

Ovarian torsion is determined when an ovary becomes twisted on its ligamentous supports and can result in compromised blood supply to the ovary (1). Ovarian torsion is characterized by a twisted ovary with or without the involvement of the fallopian tube. The clinical symptoms of ovarian torsion are variable and nonspecific. Classic clinical symptoms include the sudden onset of severe pelvic pain and a palpable adnexal mass (2). Moreover, nausea, vomiting, and pyrexia are present in some cases (3). Reproductive age is the peak period of incidence of ovarian torsion, and approximately $70 \%$ of cases occur during this period (4). It is estimated that $12 \%$ to $18 \%$ of patients with ovarian torsion are pregnant $(5,6)$, which is similar to the rate reported in our recent study (7). However, whether pregnancy is a risk factor of ovarian torsion is still controversial $(8,9)$.

Ovarian torsion is the fifth most common gynecological emergency requiring surgery, with an estimated prevalence of about $2.7 \%$ to $3 \%$ (10). Pelvic ultrasonic examination is the most frequently performed preoperative examination, but ultrasonic findings are nonspecific, despite the ultrasonic features of ovarian torsion being well-characterized $(1,11,12)$. The diagnosis of ovarian torsion can only be confirmed by surgery. Furthermore, the female reproductive function may be negatively affected by delayed treatment because of uncertainty in the preoperative diagnosis.

Ovarian torsion during pregnancy has usually been described in reports of sporadic cases (13-16). A few series of studies have focused on ovarian torsion during pregnancy or have compared ovarian torsion in pregnant and nonpregnant women $(6,9,17,18)$. Whether there are differences in the ultrasonic features and histological diagnosis of ovarian torsion among pregnant and non-pregnant women is still unclear, but a better characterization of ovarian torsion may be helpful in improving the accuracy of preoperative diagnosis.

This study aimed to compare the ultrasonic features in the preoperative diagnosis of ovarian torsion in pregnant and non-pregnant women and to describe the histological spectrum and outcomes in the two groups.

\section{Methods}

This was a retrospective investigation of data obtained for female reproductive patients with ovarian torsion at the First Affiliated Hospital of Sun Yat-sen University, a tertiary center of gynecology and obstetrics, between January 2010 and May 2017. Each patient received a detailed pelvic ultrasound examination by an experienced sonographer, including one for the uterus and adnexa. The definitive diagnosis of ovarian torsion was confirmed by surgery. Each sonographer was specialized in obstetrics and gynecology and had at least 5 years of experience performing obstetric and gynecological ultrasonic diagnoses. The Institutional Review Board of the First Affiliated Hospital of Sun Yat-sen University approved the study.

A cohort of surgically confirmed cases was divided into 2 groups according to pregnancy status. Group 1 included intrauterine pregnant women, and Group 2 included nonpregnant women who were over 18 years of age and were not postmenopausal. Group 1 was further subdivided into 2 groups based on the method of successful conception: Group 1a included intrauterine pregnant women who underwent assisted reproduction treatment; while Group 1b included natural pregnancies. The observed ultrasound features in the diagnosis of ovarian torsion were investigated. The ovarian diameter, histological diagnosis, and outcome of ovarian torsion were described. The presence of ovarian neoplasm was compared between pregnant and non-pregnant women. Ovarian cysts were regarded as a non-neoplastic lesion.

Sonography was performed using a transabdominal, transvaginal, or combined approach as appropriate using a GE Voluson730 Expert system with a 5-9-MHz transvaginal probe or an abdominal $4-8-\mathrm{MHz}$ probe (GE Healthcare, Kretztechnik, Zipf, Austria).

The ultrasonic features defining ovarian torsion were unilateral ovarian enlargement (ovarian diameter $>4 \mathrm{~cm}$ ) with or without an ovarian mass (Figure 1); ovarian edema with the presence of a hyperechogenic ovary and peripherally displaced follicles with echogenic stroma (Figure 1); abnormal adnexal position; free fluid in the Douglas pouch; the absence/decreased blood flow in the ovary, as demonstrated using Doppler ultrasound (Figure 1); and the presence of coiled, twisted, or circular (whirlpool sign) vessels (Figure 2) (1,11).

The ultrasonic features of intrauterine pregnancy included intrauterine gestational sac containing a yolk sac; empty intrauterine gestational sac with a mean diameter of $\geq 20 \mathrm{~mm}$; intrauterine gestational sac containing embryo/ fetus with or without cardiac activity (19). One of the above ultrasound criteria was required for a diagnosis of intrauterine pregnancy.

The patient ages were described as median value, while 

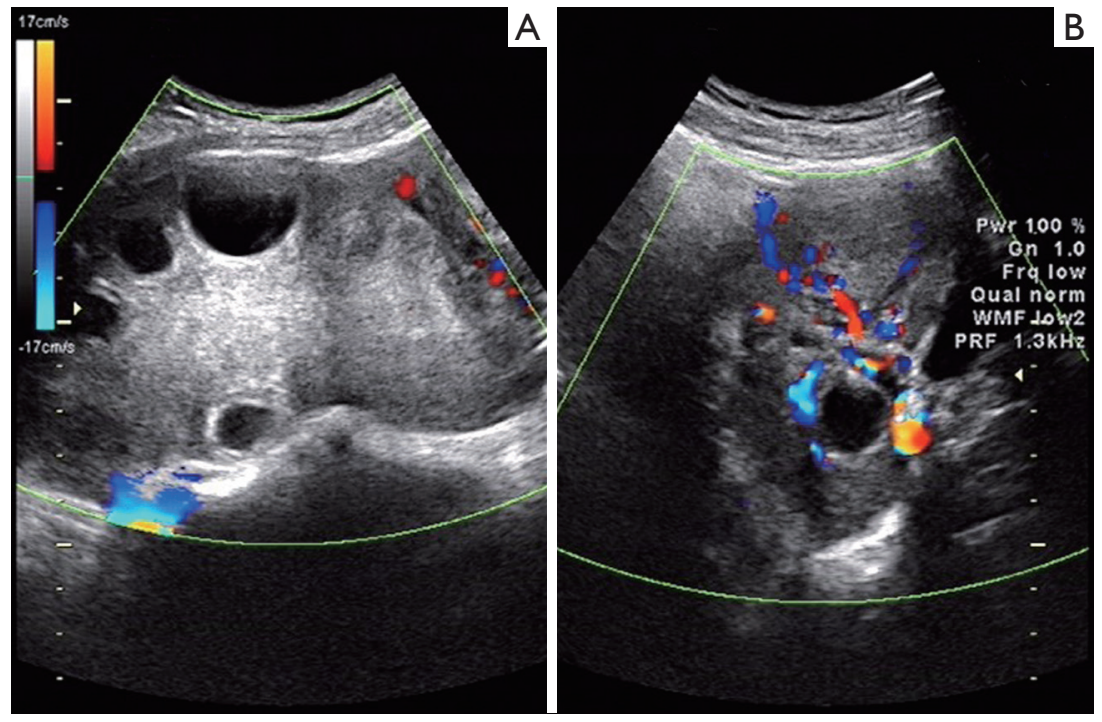

Figure 1 Trans-abdominal sonogram indicates unilateral ovary in adnexal torsion. (A) an enlarged ovary (ovarian diameter $>4 \mathrm{~cm}$ ) with ovarian edema is visible. The ovary presents hyperechogenicity, and blood flow is absent on Doppler; (B) normal-appearing contra lateral ovary.

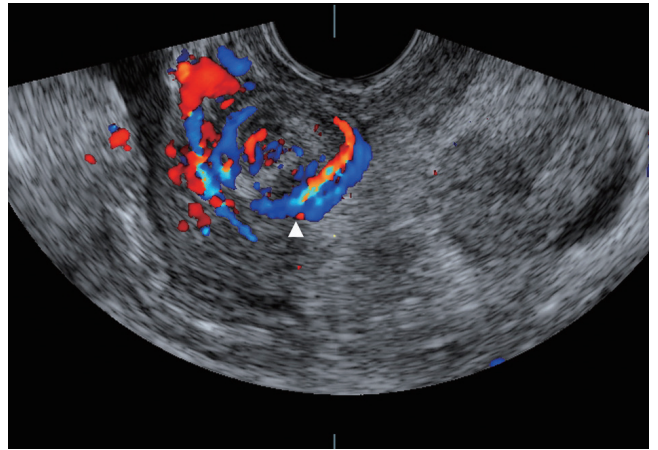

Figure 2 Transvaginal sonogram with $3 \mathrm{D}$ render mode indicates a whirlpool sign. The whirlpool sign $(\mathbf{\Delta})$ presents as coiled, twisted, or circular vessels on Doppler.

the mean and standard deviation (SD) were calculated for diameters of the twisted ovary. The median age of the patients was compared between the different groups using the Kolmogorov-Smirnov $\mathrm{Z}$ test. The diameters of the twisted ovary were compared between the different groups using the $t$-test. The twisted sides were compared between the different groups using the Chi-square test. The detection rates of preoperative ultrasound were calculated for ovarian torsion and were compared between the different groups using Fisher's exact test. The ultrasonic features of ovarian torsion in different groups were compared using Chi-square tests or Fisher's exact test. The presence of ovarian neoplasm was compared between the different groups using the Chi-square test. Results were considered statistically significant at $\mathrm{P}<0.05$. Statistical analyses were performed using SPSS 13.0 statistical software for Windows (SPSS Inc., Chicago, IL, USA).

\section{Results}

A total of 109 women with ovarian torsion at reproductive age were included in this study: 25 were pregnant women, and 84 were non-pregnant women. Examples of sonographic images of pregnant and non-pregnant women are shown in Figures 3,4. The clinical characteristics of ovarian torsion in pregnant women and non-pregnant women are listed in Table 1 . The median age of all patients was 27 [18-53] years. There were no significant differences in the median patient ages between Group 1 and Group 2 $(\mathrm{P}=0.24)$. The average diameter of the twisted ovaries of all patients was $101.4 \pm 33.1 \mathrm{~mm}$. There was no significant difference in the mean ovarian diameter between Group 1 and Group $2(\mathrm{P}=0.12)$. Forty-eight cases presented ovaries twisted on the left, whereas in 61 cases, the ovaries were twisted on the right. There was no significant difference in the twisted side between Groups 1 and $2(\mathrm{P}=0.36)$. The clinical symptoms of ovarian torsion included the sudden 

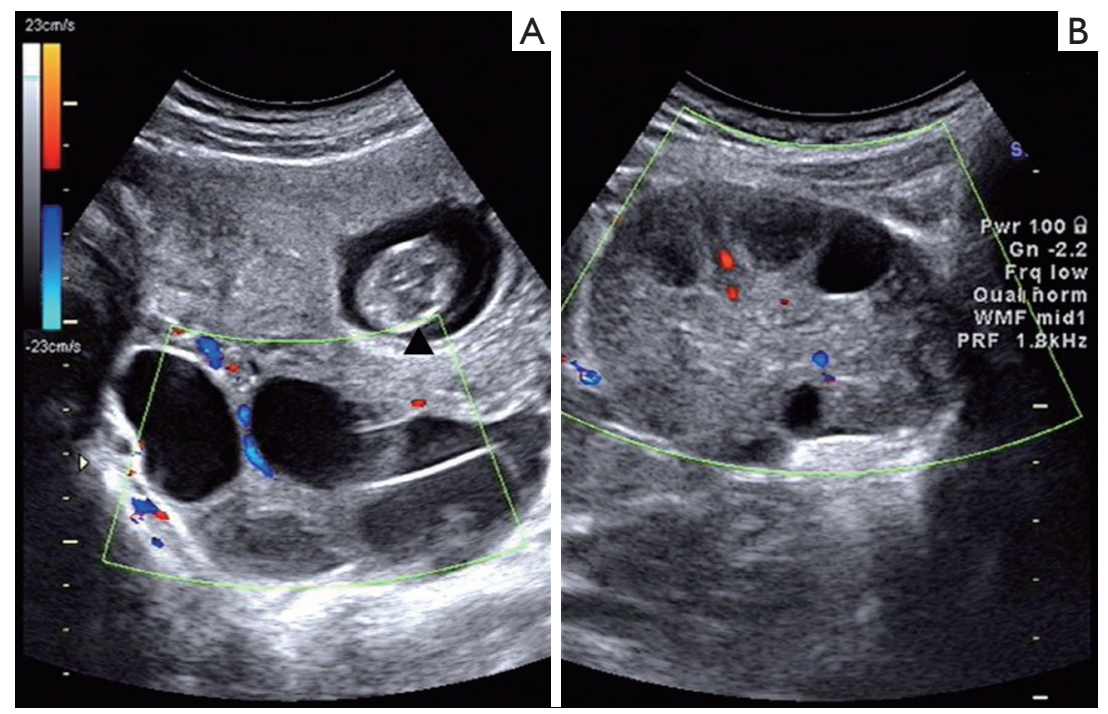

Figure 3 Ovarian torsion occurring in a 24-year-old pregnant woman with severe left lower abdominal pain for 1 day at gestational week 11. The patient underwent ovulation induction. (A) The trans-abdominal sonogram indicates an enlarged right ovary because of ovulation induction and the fetus ( $\boldsymbol{\Delta}$ indicates the fetal head); (B) the trans-abdominal sonogram indicates an enlarged left ovary with ovarian torsion. The ovary was hyperechogenic, and blood flow was absent on Doppler. Intra-operative findings confirmed that the ovary and tube twisted $360^{\circ}$.
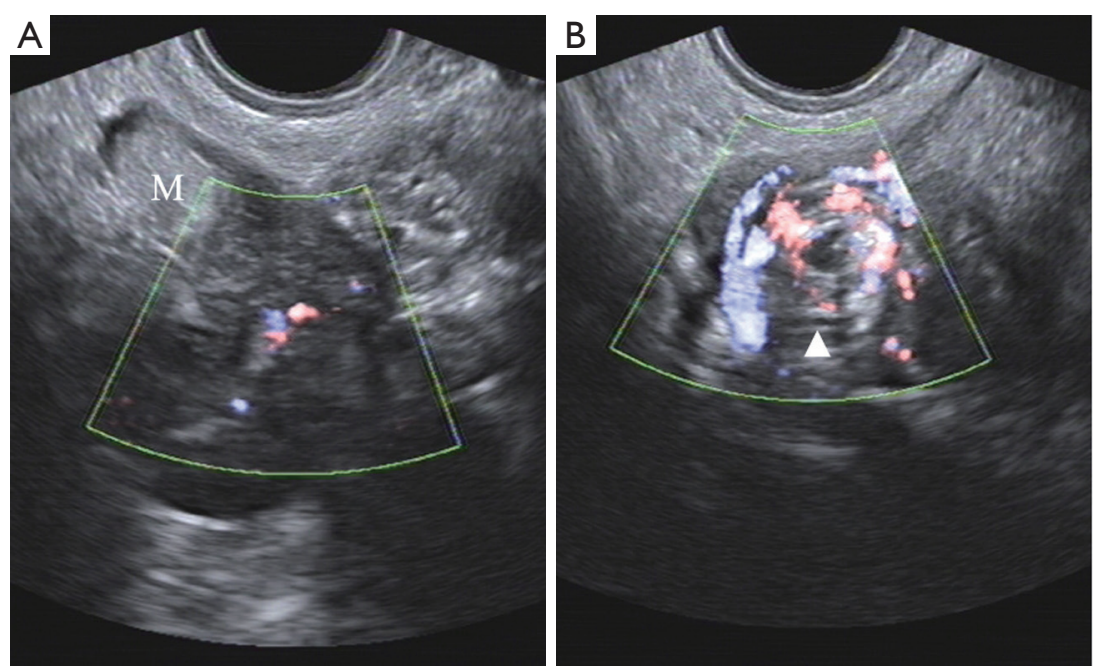

Figure 4 Ovarian torsion occurring in a 26-year-old non-pregnant woman with severe right lower abdominal pain for 1 day. (A) Transvaginal sonogram indicates an enlarged right ovary with teratoma ( $M$ : indicates ovarian teratoma), and the ipsilateral residual ovary was edematous with decreased blood flow on Doppler; (B) the transvaginal sonogram indicates a whirlpool sign ( $\mathbf{\Delta})$ on Doppler. Intraoperative findings confirmed that the ovary and tube twisted $720^{\circ}$.

onset of severe pelvic pain, a palpable adnexal mass, pyrexia, nausea, and vomiting. The occurrence of palpable adnexal mass was significantly different between Group 1 and Group 2 ( $\mathrm{P}=0.02)$. There were no significant differences in the other clinical symptoms between Group 1 and Group 2.

Of the 109 cases of ovarian torsion presenting at reproductive age, preoperative ultrasonography was confirmed by the surgical findings in 91 cases and not 
Table 1 Clinical characteristics of ovarian torsion in pregnant and non-pregnant women

\begin{tabular}{|c|c|c|c|c|}
\hline Clinical characteristics & Group $1(n=25)$ & Group $2(n=84)$ & Total $(n=109)$ & $\mathrm{P}$ \\
\hline Torsion side & & & & $0.36^{\ddagger}$ \\
\hline Left & 9 (36.0\%) & $39(46.4 \%)$ & $48(44.0 \%)$ & \\
\hline Right & $16(64.0 \%)$ & 45 (53.6\%) & $61(56.0 \%)$ & \\
\hline \multicolumn{5}{|l|}{ Symptoms } \\
\hline Sudden onset of severe pelvic pain & $25(100 \%)$ & $76(90.5 \%)$ & $101(92.7 \%)$ & $0.19^{\ddagger}$ \\
\hline A palpable adnexal mass & $1(4.0 \%)$ & $22(26.2 \%)$ & $23(21.1 \%)$ & $0.02^{\ddagger}$ \\
\hline Pyrexia & $0(0)$ & $3(3.6 \%)$ & $3(2.8 \%)$ & $1.00^{\ddagger}$ \\
\hline Nausea and vomiting & $1(4.0 \%)$ & $7(8.3 \%)$ & $8(7.3 \%)$ & $0.68^{\ddagger}$ \\
\hline
\end{tabular}

Group 1, intrauterine pregnant women; Group 2, non-pregnant women. ${ }^{\dagger}$, comparisons were assessed using the Kolmogorov-Smirnov $Z$ test; ${ }^{\ddagger}$, comparisons were assessed with Fisher's exact tests; ${ }^{\S}$, comparisons were assessed with $t$-test.

confirmed in 18 cases. The overall detection rate of preoperative ultrasound for all patients was 0.84 . The detection rates of preoperative ultrasound in Group 1 and Group 2 were 1.00 and 0.79 , respectively. There was a significant difference in the detection rates of preoperative ultrasound between Groups 1 and $2(\mathrm{P}=0.01)$. The detection rate of preoperative ultrasound in pregnant women was higher than in non-pregnant women. Clinical characteristics in the matched and the missed cases of ovarian torsion at reproductive ages are listed in Table 2. Unilateral ovarian enlargement occurred in all the matched cases and in all the missed cases. Seventeen (94.4\%) missed cases presented neoplastic lesions, and only one case had a corpus luteum. There were significant differences in the presence of ovarian edema $(\mathrm{P}<0.001)$, absence/decreased blood flow in the ovary $(\mathrm{P}=0.01)$, and the whirlpool sign $(\mathrm{P}<0.001)$ between the matched and missed cases. There were no significant differences regarding the presence of abnormal adnexal positions $(\mathrm{P}=0.23)$ and free fluid in the pouch of Douglas $(\mathrm{P}=0.93)$ between matched and missed cases. There was a significant difference in the twisted side involved between the matched and the missed cases $(\mathrm{P}=0.002)$.

The presence of ultrasonic hallmarks in the different groups is listed in Table 3. Unilateral ovarian enlargement occurred in all cases of ovarian torsion. Ovarian edema, abnormal adnexal positions, free fluid in the pouch, absence/decreased blood flow in the ovary, and the whirlpool sign occurred in 55, 22, 22, 73, and 65 cases, respectively. There were significant differences in the presence of ovarian edema $(\mathrm{P}<0.01)$ and abnormal adnexal positions $(\mathrm{P}=0.02)$ between Groups 1 and 2. There were no significant differences in the presence of free fluid in the pouch of Douglas $(\mathrm{P}=0.25)$, the absence/decreased blood flow in the ovary $(\mathrm{P}=0.40)$, or the Whirlpool sign $(\mathrm{P}=0.06)$ between Groups 1 and 2.

The patients were treated via adnexectomy or conservative surgery. Adnexectomy was performed to remove the affected ovary and fallopian tube. Conservative surgery involved detorsion of the adnexa with or without lesionectomy. Adnexectomy was performed in 62 (56.9\%) patients, and conservative surgery was performed in 47 (43.1\%) patients. The surgical procedures are listed in Table 4. None of the patients experienced any other postoperative complications. The histological diagnoses are listed as follows in descending order: teratoma (38/109), ovarian cyst (30/109), ovary (22/109), epithelial tumor (15/108), and sex cord stromal tumor (4/109). According to the histological classification of the WHO (2014), ovarian cysts are regarded as non-neoplastic lesions. In total, there were 52 non-neoplastic cases and 57 neoplastic cases in our cohort. Of the non-neoplastic cases, 38 underwent adnexectomy, and 14 underwent conservative surgery. Of the neoplastic cases, 24 patients underwent adnexectomy, and 33 patients underwent conservative surgery. There were significant differences in the surgical procedures used for Groups 1 and 2 with neoplastic or non-neoplastic lesions $(\mathrm{P}=0.002)$. The histological diagnoses of Group 1 are listed as follows in descending order: ovary $(10 / 25)$, ovarian cyst 
Table 2 Clinical findings in the matched and the missed cases of ovarian torsion at reproductive age

\begin{tabular}{|c|c|c|c|}
\hline Clinical characteristics & Matched, n (\%) & Missed, n (\%) & $P$ value \\
\hline Group 1 & $25(100.0)$ & $0(0.0)$ & \\
\hline Group 1a & $8(100.0)$ & $0(0.0)$ & \\
\hline Group 1b & $17(100.0)$ & $0(0.0)$ & \\
\hline \multicolumn{4}{|l|}{ Ultrasound findings } \\
\hline Unilateral ovarian enlargement & $91(100.0)$ & $18(100.0)$ & $-^{\ddagger}$ \\
\hline Ovarian edema & $55(60.4)$ & $0(0.0)$ & $<0.001^{\dagger}$ \\
\hline Abnormal adnexal position & $16(17.6)$ & $6(33.3)$ & $0.23^{\dagger}$ \\
\hline Whirlpool sign & $64(70.3)$ & $1(5.6)$ & $<0.001^{\dagger}$ \\
\hline Torsion side & 91 & 18 & $0.002^{\dagger}$ \\
\hline Left & $34(37.4)$ & $14(77.8)$ & \\
\hline Right & $57(62.6)$ & $4(22.2)$ & \\
\hline
\end{tabular}

Group 1, intrauterine pregnant women; Group 2, non-pregnant women. ${ }^{\ddagger}$, unilateral ovarian enlargement/adnexal mass occurred in all cases; thus, no statistics were computed; ${ }^{\dagger}$, comparisons were assessed by Chi-square test.

Table 3 Ultrasound findings of ovarian torsion in pregnant women and non-pregnant women

\begin{tabular}{|c|c|c|c|}
\hline Ultrasound findings & Group 1, n (\%) & Group 2, n (\%) & $\mathrm{P}$ value \\
\hline Ovarian edema & $19(76.0)$ & $36(42.9)$ & $<0.01^{\ddagger}$ \\
\hline Abnormal adnexal position & $1(4.0)$ & $21(0.25)$ & $0.02^{\ddagger}$ \\
\hline Free fluid in the pouch of Douglas & $3(12.0)$ & $19(22.6)$ & $0.25^{\ddagger}$ \\
\hline Whirlpool sign & $19(76.0)$ & $46(54.8)$ & $0.06^{\ddagger}$ \\
\hline
\end{tabular}

Group 1, intrauterine pregnant women; Group 2, non-pregnant women. ${ }^{\dagger}$, unilateral ovarian enlargement occurred in all cases; thus, no statistics were computed; ${ }^{\ddagger}$, comparisons were assessed by Chi-square test. Whirlpool sign refers to coiled, twisted, or circular vessels.

(9/25), teratoma (3/25), and epithelial tumor (3/25). The histological diagnoses of Group 2 are listed as follows in descending order: teratoma (35/84), ovarian cyst $(21 / 84)$, ovary (12/84), epithelial tumor (12/84), and sex cord stromal tumor (4/84). The histological diagnoses are listed in Table 5 . Group 2 had a significantly higher number of ovarian neoplasms than Group $1(\mathrm{P}=0.001)$.

In Group 1, 18 cases $(72.0 \%, 18 / 25)$ occurred in the first trimester, $5(20.0 \%, 5 / 25)$ cases occurred in the second trimester, and $2(8.0 \%, 2 / 25)$ cases occurred in the third trimester. The clinical characteristics of ovarian torsion in pregnant women are listed in Table 6. Eight cases (Group 1a) in Group 1 received assisted reproduction treatment, and 17 cases (Group 1b) were natural pregnancies. In the first trimester, 3 cases underwent in vivo fertilization and embryo transfer, and 3 cases underwent ovulation induction. In the second trimester, 1 case underwent fertilization in vitro and embryo transfer. In the third trimester, 1 case underwent 
Table 4 Treatment of ovarian torsion in pregnant women and non-pregnant women

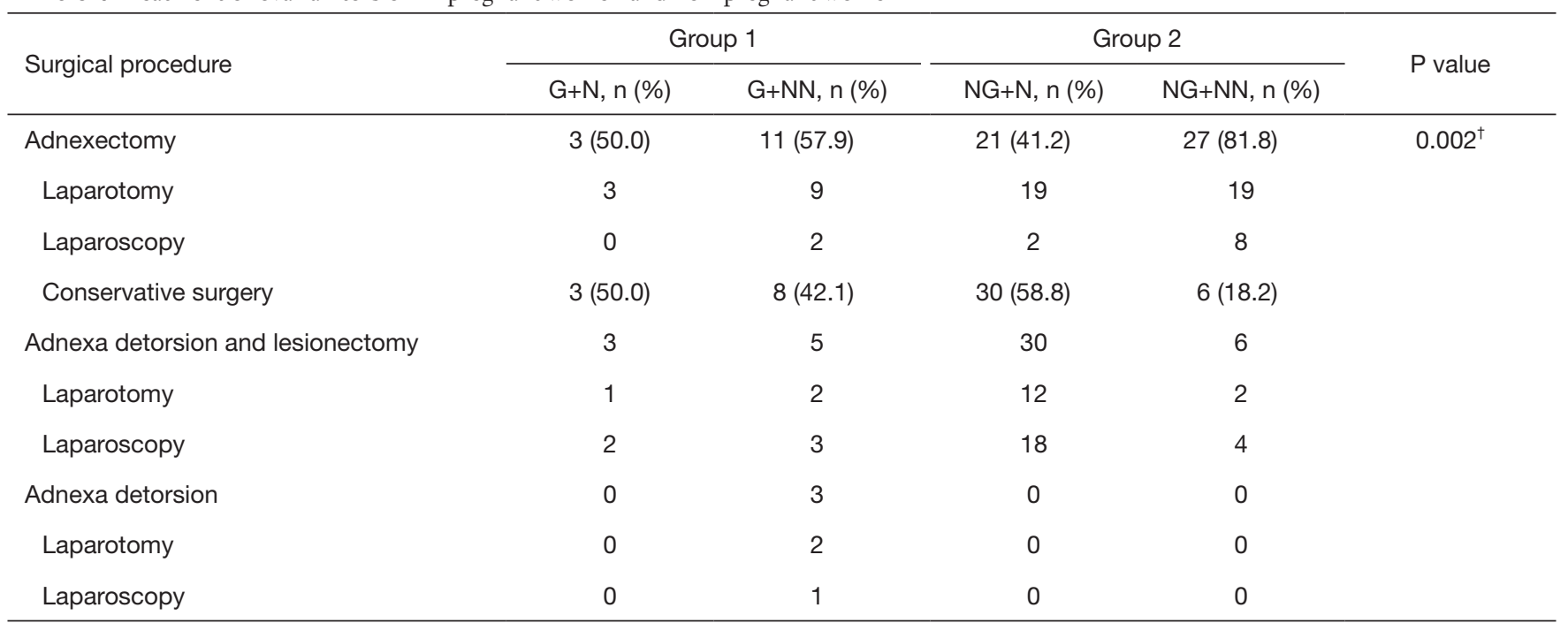

Group 1, intrauterine pregnant women; Group 2, non-pregnant women. G+N, intrauterine pregnant women with ovarian neoplastic lesion; $\mathrm{G}+\mathrm{NN}$, intrauterine pregnant women with ovarian non-neoplastic lesion; NG+N, non-pregnant women with ovarian neoplastic lesion; $\mathrm{NG}+\mathrm{NN}$, non-pregnant women with ovarian non-neoplastic lesion. ${ }^{\dagger}$, comparisons were assessed with Fisher's exact tests.

Table 5 Histologic diagnosis of ovarian torsion in pregnant women and non-pregnant women

\begin{tabular}{lcc}
\hline Histologic diagnosis [n] & Group 1 & Group 2 \\
\hline Germ cell tumor [38] & 3 & 35 \\
Mature teratoma [38] & & \\
Epithelial tumor [15] & 1 & 2 \\
Serous cystadenoma [3] & 0 & 2 \\
Serous carcinoma [2] & 1 & 7 \\
Mucinous cystadenoma [8] & 1 & 0 \\
Borderline mucinous & & 1 \\
cystadenoma [1] & 0 & \\
Benign Brenner tumor [1] & & 1 \\
Sex cord-stromal tumor [4] & 0 & 12 \\
Thecoma [2] & 0 & 34 \\
Fibroma [1] & 0 & 12 \\
Thecofibroma [1] & 10 & \\
Ovary [22] & 5 & \\
Ovarian cyst [30] & 5 & \\
Simple cyst [23] & 5 & \\
Hematoma [7] & & 18 \\
\hline Total & & \\
\hline
\end{tabular}

Group 1, intrauterine pregnant women; Group 2, non-pregnant women. fertilization in vitro and embryo transfer. None of the cases in Group 2 received assisted reproduction treatment. There were significant differences in the torsion side, symptoms, occurrence time of ovarian torsion, ovarian diameter, and ultrasound findings between Group 1a and Group 1b.

\section{Discussion}

Ovarian torsion is a gynecologic surgical emergency, involving partial or total rotation of the ovary about its vascular axis. This has the potential to impede venous or lymphatic drainage, which in turn can result in congestion of the ovarian parenchyma and reduction or cessation of arterial perfusion, which leads to infarction, hemorrhagic necrosis, and gangrene (20). It has been reported that delayed diagnosis possibly leads to serious complications, including the loss of the adnexa or ovary, fatal thrombophlebitis, or peritonitis (21). The incidence of ovarian torsion during pregnancy is estimated to occur in 1 to 5:10,000 pregnancies (18). However, there have only been a few series studies that have compared the clinical characteristics and treatment of ovarian torsion in pregnant and non-pregnant women $(8,17,18)$. Furthermore, the ultrasonic features and the histological diagnosis of ovarian torsion in pregnant and non-pregnant women have not been described, and are thus the focus of our study.

Ovarian torsion during pregnancy occurs in about $12 \%$ 
Table 6 Clinical characters of ovarian torsion in pregnant women

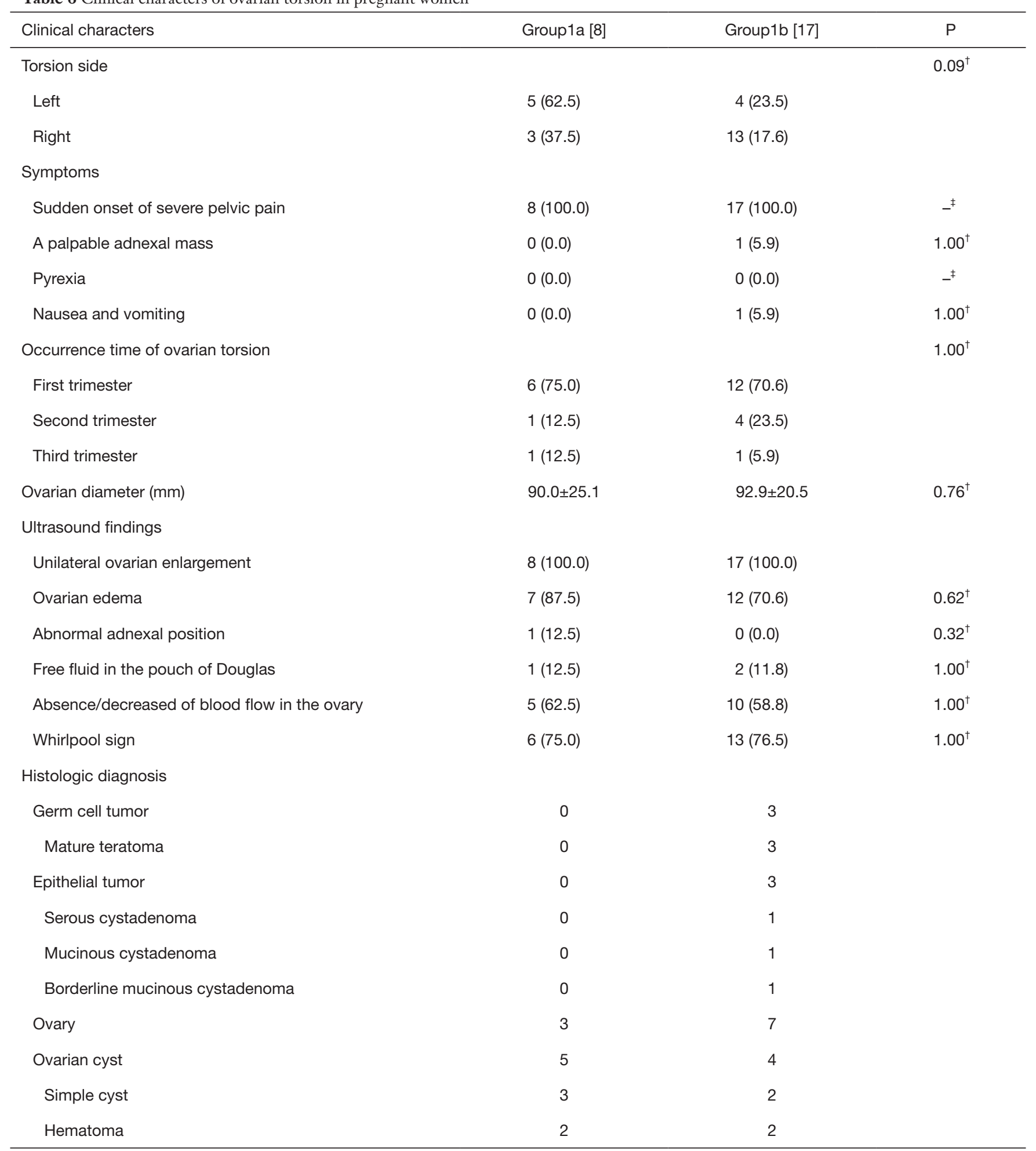

Group 1a, pregnant women who received assisted reproduction treatment; Group 1b, natural pregnant women. ${ }^{\ddagger}$, sudden onset of severe pelvic pain and pyrexia occurred in all cases; thus, no statistics were computed; ${ }^{\dagger}$, comparisons were assessed by Fisher's exact tests; ${ }^{\S}$, the comparisons were assessed by $t$-test. 
to $18 \%$ of cases of ovarian torsion overall $(5,6)$, which is in agreement with our data. Ovarian torsion may occur at any time during pregnancy, although it has been reported to occur more frequently in the first trimester $(13,15,16,22,23)$. Our study confirmed the findings that ovarian torsion primarily occurred in the first trimester. A third of the cases of ovarian torsion we observed during pregnancy occurred in women that had undergone assisted reproduction treatment, which supported previous reports indicating that assisted reproduction treatment was a risk factor for ovarian torsion in pregnancy $(6,17,18)$.

Furthermore, our data indicated that ovarian torsion could occur independently of reproductive age because there was no significant difference in the median age of patients comparing pregnant and non-pregnant groups.

We observed no significant difference in the mean ovarian diameter in pregnant and non-pregnant patients in our study. Furthermore, the mean ovarian diameter in patients with ovarian torsion of the assisted reproduction treatment group and the natural pregnancy group was not significantly different. This indicates that the diagnosis of ovarian torsion should be suspected when the ovarian diameter increases to approximately $10 \mathrm{~cm}$ in the presence of clinical symptoms at reproductive ages, whether or not the patient is pregnant. Our data showed that the right side was dominant in cases of ovarian torsion. This significant difference in the twisted side occurring between matched and missed cases suggests that right-sided ovarian torsion was more detectable. There was no significant difference in the twisted side between Group 1 and Group 2, which indicates that the right side was dominant in cases of ovarian torsion independently of pregnancy status. There are two possible reasons for this phenomenon: one is that the right utero-ovarian ligament is physiologically longer than the left ligament and may favor ovary rotation, and the other is due to the presence of the sigmoid colon on the left, which reduces the space available for the torsion to occur (24-26).

The clinical symptoms of ovarian torsion in our study were similar to those of a previous report $(17,18)$. The most frequent symptom was the sudden onset of severe pelvic pain, and the second was a palpable adnexal mass. Moreover, pyrexia, nausea, and vomiting occurred in some cases. The occurrence of a palpable adnexal mass was a significant difference between Groups 1 and 2. The abdominal mass in the non-pregnant group was more palpable than that of the pregnant group. This may be due to uterine enlargement during pregnancy, which affects the palpation of the adnexal mass.
Ultrasound examination plays an important role in the preoperative diagnosis of ovarian torsion. The ultrasonic features of ovarian torsion included unilateral ovarian enlargement, ovarian edema, abnormal adnexal position, free fluid in the Douglas pouch, the absence/decreased of blood flow in the ovary, and the whirlpool sign $(1,11)$, all of which are associated with the pathophysiological changes observed following ovarian torsion. When ovarian torsion occurs, ovarian venous and lymphatic reflux may be blocked, and ovarian parenchymal congestion may lead to ovarian enlargement, ovarian edema, and the accumulation of free fluid in the Douglas pouch. A decrease or interruption of ovarian arterial perfusion may lead to absence/decreased blood flow to the ovary. Ovarian torsion is the result of the rotation of the pedicle, which can cause twisting of the blood vessels in the pedicle to form the whirlpool sign. Ovarian torsion can change the position of the ovary and lead to an abnormal adnexal position. The unilateral ovarian enlargement was identified in both matched and missed cases. Most missed cases were associated with ovarian neoplastic lesions, which might have affected the residual ovarian tissue. In our study, the identification of ultrasonic hallmarks, except for unilateral ovarian enlargement, varied when the matched and the missed cases of ovarian torsion were compared. There were significant differences concerning the presence of ovarian edema, the absence/decreased of blood flow in the ovary, and the whirlpool sign between the matched and missed cases, which indicates that these could represent reliable ultrasonographic markers of ovarian torsion. It was worth noting that unilateral ovarian enlargement occurred in all cases, independently of pregnancy status, which suggests unilateral ovarian enlargement could be a diagnostic marker in clinically suspected cases. There were no significant differences concerning the presence of abnormal adnexal position and the free fluid in the pouch of Douglas of the matched and missed cases. Moreover, the missed cases were all in the non-pregnant women group, which indicates that the presence of the abnormal adnexal position and the free fluid in the pouch of Douglas did not apply to some cases, especially non-pregnant subjects.

In our study, the detection rate of preoperative ultrasound in pregnant subjects were higher than in nonpregnant subjects. There are three possible reasons for this observation. First, ovarian edema is more obvious in pregnant women and is a reliable marker of suspected ovarian torsion. Second, an enlarged uterus during pregnancy reduces pelvic space, which allows for the 
easy detection of enlarged ovaries. Third, the incidence of ovarian neoplasm is pregnant women was lower, and the ovarian neoplasm might have interfered with the observation of the residual normal ovarian tissue.

Ultrasonic features of ovarian torsion between pregnant and non-pregnant women have also been mentioned in previous studies, in which ovarian torsion was not the main focus $(6,17,18)$. A precise preoperative ultrasonic diagnosis of ovarian torsion is critical because it forms the basis of treatment.

Our study found that the presence of specific ultrasonographic features of ovarian torsion varied across the different groups. Decreased venous and lymphatic drainage resulted in ongoing ovarian tissue congestion, which led to ischemia and eventually to ovarian necrosis. Thus, ovarian enlargement and ovarian edema represent characteristic manifestations of ovarian torsion. Ovarian edema in pregnant women is more obvious than in nonpregnant women, which may be associated with the lower incidence of ovarian neoplasms in pregnant women. The ovarian neoplasm may interfere with the observation of the remaining normal ovarian tissue. An abnormal adnexal position was identified in 1 pregnant case, possibly because the enlarged uterus hindered the change in ovarian position. Furthermore, our study found that the presence of ultrasonographic features of ovarian torsion was not significantly different between Group 1a and Group 1b, which indicates that these features were similar in the pregnant group irrespective of the conception method.

The histological diagnoses of ovarian torsion between pregnant and non-pregnant women have been mentioned in a few previous studies with the most common histological diagnoses varying among studies $(6,18)$. In our cohort, the histological diagnosis varied among pregnant and nonpregnant women. For pregnant women, the most common histological diagnosis was a normal ovary, which was in contrast to that in previous studies that reported serous cysts, corpora lutea cysts, and follicular cysts as common causes of torsion $(6,18)$. Moreover, in our study, teratoma was the most common diagnosis in non-pregnant fertile women, which was consistent with previous studies (17). According to the histological classification of the WHO (2014), ovarian cysts are regarded as non-neoplastic lesions. There were significant differences in the presence of ovarian neoplasm between the pregnant and non-pregnant groups. In cases of ovarian torsion, non-neoplastic lesions were common in pregnant women, whereas neoplastic lesions were common in non-pregnant women.
There are two approaches to the management of ovarian torsion: one involves adnexectomy, which removes the affected ovary and the fallopian tube; the other is conservative surgery, which involves adnexa detorsion with or without lesionectomy $(3,24,27)$. When the adnexa appears necrotic or appears unviable after detorsion of the ischemic adnexa, adnexectomy is generally the preferred approach (3). Recently, conservative surgery has been advocated to protect ovarian function $(24,27)$. Our data suggest that the proportion of ovarian necrosis in nonneoplastic cases was higher than that in neoplastic cases; thus, adnexectomy was more common in non-neoplastic cases in our cohort.

Our study has some limitations. The retrospective study design and the exclusion from the study of those patients not undergoing operation might have resulted in the exclusion of more patients with ovarian torsion, limiting the generalizability of our results. Despite these limitations, we can conclude from our study that ovarian edema, the absence/decreased of blood flow in the ovary, and the whirlpool sign were reliable ultrasonographic markers of ovarian torsion. A diagnosis of ovarian torsion should be suspected when the ovary is enlarged and in the presence of clinical symptoms in patients of reproductive age irrespective of pregnancy status. The preoperative ultrasonic detection rate of ovarian torsion in pregnant women was higher than in non-pregnant women. Moreover, the presence of ovarian edema in pregnant women was more pronounced than in non-pregnant women. Finally, the ultrasonographic features of ovarian torsion were similar and did not differ according to the method of conception. In the cases of ovarian torsion, non-neoplastic lesions were more common in pregnant women, whereas neoplastic lesions were common in nonpregnant women.

\section{Acknowledgments}

None.

\section{Footnote}

Conflicts of Interest: The authors have no conflicts of interest to declare.

Ethical Statement: The Institutional Review Board of the First Affiliated Hospital of Sun Yat-sen University approved the study. 


\section{References}

1. Chang HC, Bhatt S, Dogra VS. Pearls and pitfalls in diagnosis of ovarian torsion. Radiographics 2008;28:1355-68.

2. Nair S, Joy S, Nayar J. Five year retrospective case series of adnexal torsion. J Clin Diagn Res 2014;8:OC09-13.

3. Huchon C, Fauconnier A. Adnexal torsion: a literature review. Eur J Obstet Gynecol Reprod Biol 2010;150:8-12.

4. Erdemoğlu M, Kuyumcuoglu U, Guzel AI. Clinical experience of adnexal torsion: evaluation of 143 cases. J Exp Ther Oncol 2011;9:171-4.

5. Houry D, Abbott JT. Ovarian torsion: a fifteen-year review. Ann Emerg Med 2001;38:156-9.

6. Bider D, Mashiach S, Dulitzky M, Kokia E, Lipitz S, BenRafael Z. Clinical, surgical and pathologic findings of adnexal torsion in pregnant and nonpregnant women. Surg Gynecol Obstet 1991;173:363-6.

7. Feng JL, Lei T, Xie HN, Li LJ, Du L. Spectrums and Outcomes of Adnexal Torsion at Different Ages. J Ultrasound Med 2017;36:1859-66.

8. Oelsner G, Shashar D. Adnexal torsion. Clin Obstet Gynecol 2006;49:459-63.

9. Yuk JS, Shin JY, Park WI, Kim DW, Shin JW, Lee JH. Association between pregnancy and adnexal torsion: A population-based, matched case-control study. Medicine 2016;95:e3861.

10. Mashiach R, Melamed N, Gilad N, Ben-Shitrit G, Meizner I. Sonographic diagnosis of ovarian torsion accuracy and predictive factors. J Ultrasound Med 2011;30:1205-10.

11. Valsky DV, Esh-Broder E, Cohen SM, Lipschuetz M, Yagel S. Added value of the gray-scale whirlpool sign in the diagnosis of adnexal torsion. Ultrasound Obstet Gynecol 2010;36:630-4.

12. Gerscovich EO, Corwin MT, Sekhon S, Runner GJ, Gandour-Edwards RF. Sonographic appearance of adnexal torsion, correlation with other imaging modalities, and clinical history. Ultrasound Q 2014;30:49-55.

13. Passarinho RM, Afonso E, Reis L, Santos Silva I. Adnexal torsion in third trimester of pregnancy. BMJ Case Rep 2012;2012. doi: 10.1136/bcr-2012-006755.

14. Morton MJ, Masterson M, Hoffmann B. Case report: ovarian torsion in pregnancy - diagnosis and management. J Emerg Med 2013;45:348-51.

15. Kahramanoglu I, Eroglu V, Turan H, Kaval G, Sal $\mathrm{V}$, Tokgozoglu N. Isolated adnexal torsion in a 20week spontaneous twin pregnancy. Int J Surg Case Rep
2016;23:138-40.

16. Aydin T, Yucel B. Laparoscopic management of adnexal torsion in a twin, in vitro fertilization pregnancy at 23 weeks. Wideochir Inne Tech Maloinwazyjne 2014;9:655-7.

17. Hasson J, Tsafrir Z, Azem F, Bar-On S, Almog B, Mashiach R, Seidman D, Lessing JB, Grisaru D. Comparison of adnexal torsion between pregnant and nonpregnant women. Am J Obstet Gynecol 2010;202:536.e1-6.

18. Ginath S, Shalev A, Keidar R, Kerner R, Condrea A, Golan A, Sagiv R. Differences between adnexal torsion in pregnant and nonpregnant women. J Minim Invasive Gynecol 2012;19:708-14.

19. Farquharson RG, Jauniaux E, Exalto N. Updated and revised nomenclature for description of early pregnancy events. Hum Reprod 2005;20:3008-11.

20. Bharathan R, Ramsawak L, Kelly A. Ovarian torsion: opportunities to improve clinical management. J Obstet Gynaecol 2012;32:683-6.

21. Bayer AI, Wiskind AK. Adnexal torsion: can the adnexa be saved? Am J Obstet Gynecol 1994;171:1516-10.

22. Tsai HC, Kuo TN, Chung MT, Lin MY, Kang CY, Tsai YC. Acute abdomen in early pregnancy due to ovarian torsion following successful in vitro fertilization treatment. Taiwan J Obstet Gynecol 2015;54:438-41.

23. Li Q, Li X, Zhang P. Ovarian torsion caused by hyperreactio luteinalis in the third trimester of pregnancy: a case report. Int J Clin Exp Med 2015;8:19612-5.

24. Ekin M, Ya§ar L, Özdemir IA, Idil S. The management of adnexal torsion: ovaries can be saved by early diagnostic laparoscopy and detorsion. Bakırköy Tip Der 2011;7:125-9.

25. Pena JE, Ufberg D, Cooney N, Denis AL. Usefulness of Doppler sonography in the diagnosis of ovarian torsion. Fertil Steril 2000;73:1047-50.

26. Warner MA, Fleischer AC, Edell SL, Thieme GA, Bundy AL, Kurtz AB, James AE Jr. Uterine adnexal torsion: sonographic findings. Radiology 1985;154:773-5.

27. Erkal N, İsenlik BS, Çağlar M, Sahillioğlu B, Kumru S. Management of adnexal torsion. J Clin Exp Invest 2014;5:7-11.

Cite this article as: Feng JL, Zheng J, Lei T, Xu YJ, Pang $\mathrm{H}$, Xie HN. Comparison of ovarian torsion between pregnant and non-pregnant women at reproductive ages: sonographic and pathological findings. Quant Imaging Med Surg 2020;10(1):137-147. doi: 10.21037/qims.2019.11.06 